\title{
Erektile Dysfunktion: So klappt es mit der Therapie
}

\begin{abstract}
Spricht ein Patient mit erektiler Dysfunktion schlecht auf eine Therapie mit PDE-5-Hemmer an, kann es an der Dosis liegen - oder der Mann wendet das Medikament falsch an. Manchmal liegt ein Hypogonadismus vor.
\end{abstract}

Erst wenn länger als ein halbes Jahr dauerhaft die Unfähigkeit besteht, eine für befriedigenden Geschlechtsverkehr ausreichende Erektion aufzubauen oder zu erhalten, wird definitionsgemäß von einer erektilen Dysfunktion (ED) gesprochen. In den meisten Fällen liegt eine organische Ursache vor. Psychologische und interpersonelle Faktoren sind ebenfalls nicht zu vernachlässigende Ursachen.

\section{Genaue Diagnostik wichtig}

Für eine vollständige Erektion sind neurogene, glattmuskuläre, arterielle, venöse und hormonelle Körperfunktionen wichtig. Daran erinnert der Freiburger Urologe Dr. Christian Leiber [1]. Dementsprechend wichtig ist die genaue Diagnostik vor Beginn einer Therapie. Für die ED und kardiovaskuläre Erkrankungen seien grundsätzlich die gleichen Risikofaktoren maßgeblich, schreibt Leiber. „Erektionsstörungen können daher als Warnsignal für ein erhöhtes kardiovaskuläres Risiko bei Männern angesehen werden“, betont er und mahnt eine entsprechend gründli- che diagnostische Klärung an. Des Weiteren verschlechtern eine ganze Reihe von Medikamenten die erektile Funktion, etwa nichtselektive Betablocker, Thiaziddiuretika, trizyklische Antidepressiva, Methyldopa und Benzodiazepine.

Bei der klinischen Untersuchung sollte der Arzt laut Leiber auf Zeichen eines Testosteron-Mangelsyndroms wie Gynäkomastie, fehlende Behaarung oder eine suprapubische Fettschürze achten. Er empfiehlt außer der orientierenden kardiovaskulären Untersuchung auch das Tasten der Femoralpulse. An Laboruntersuchungen empfiehlt die Europäische Gesellschaft für Urologie (EAU) in ihrer Leitlinie zur ED den Nüchternblutzucker, die Nüchternblutfette sowie den Gesamttestosteronspiegel zu bestimmen. Spezielle Untersuchungen des Penis wie die Schwellkörperinjektionstestung oder Duplexsonografie sind Spezialisten vorbehalten.

„Die Erfolgsraten sind in den meisten Fällen hoch“, so Leiber mit Blick auf die therapeutischen Optionen. Die EAU schlägt dafür einen Algorithmus vor.
Bei erektiler Dysfunktion können auch psychologische und interpersonelle Faktoren relevant sein.
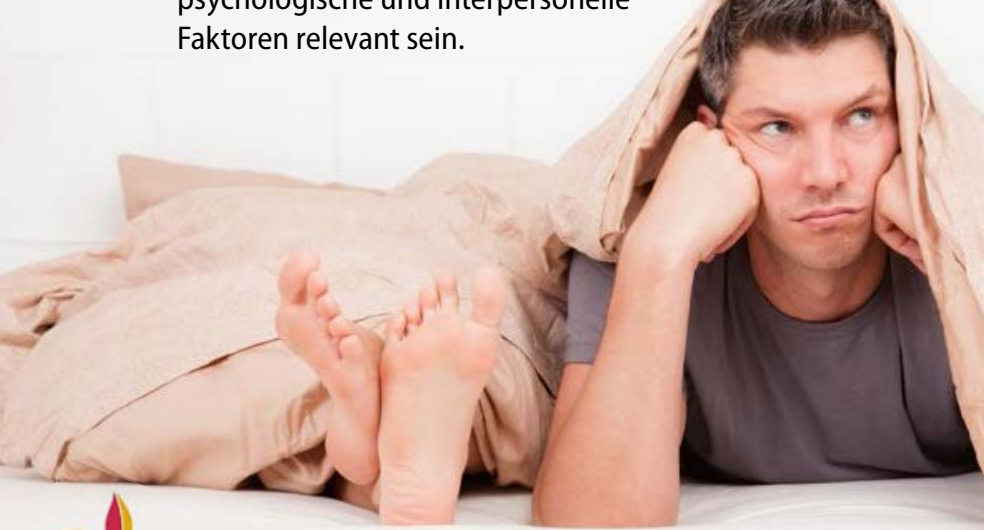

Zunächst werden behandelbare Ursachen der ED adressiert, Lebensstiländerungen angeregt, Risikofaktoren möglichst beseitigt sowie Patient und Partner/in beraten.

\section{PDE-5-Inhibitoren sind erste Wahl}

In puncto medikamentöser Therapie stellen Phosphodiesterase-5-Inhibitoren (PDE-5-Hemmer) die erste Wahl dar, solange keine Kontraindikationen vorliegen. Jedoch sollen die Patienten darauf hingewiesen werden, dass ausreichende Appetenz und sexuelle Stimulation des Patienten zwingende Voraussetzungen für die Wirksamkeit dieser Substanzen sind.

Zur Verfügung stehen Sildenafil mit einer empfohlenen Startdosis von $50 \mathrm{mg}$, Tadalafil und Vardenafil mit Startdosen von jeweils $10 \mathrm{mg}$ sowie Avanafil, das anfangs mit $100 \mathrm{mg}$ dosiert werden soll. Die Wirkstoffe unterscheiden sich teilweise in der Geschwindigkeit des Wirkeintritts und der Wirkdauer, was differenzialtherapeutisch genutzt werden kann.

Erreicht der Patient kein zufriedenstellendes Ergebnis, wird zunächst die Anwendung der verordneten Therapie überprüft und der Patient erneut beraten. Häufig würden zu wenige Versuche mit zu geringer Dosis vorgenommen, erkärt Leiber. „Erst nach mehr als achtmaliger Gabe bis zur Höchstdosis kann ein wirkliches Nichtansprechen diagnostiziert werden."Dann sind alternative Therapieversuche oder Kombitherapien angezeigt.

Als ein weiterer Grund für ein Therapieversagen kommt ein nicht erkannter Hypogonadismus in Betracht. Bei diesen Patienten verbessert die Substitution von Testosteron das Ansprechen auf PDE5-Hemmer. Der Freiburger Urologe rät außerdem, die Patienten explizit darauf hinzuweisen, dass PDE-5-Inhibitoren zu den weltweit am häufigsten gefälschten und über das Internet vertriebenen Medikamenten gehören.

Thomas Meissner

Urologe 2017; 56: 519-529 